\title{
Winnicott : libido précoce et sexuel profond.
}

\section{Dominique Scarfone}

" Je me rends compte moi-même que lorsque je parle de la régression et des problèmes infantiles précoces, les gens pensent très facilement que je suis incapable de faire un bout d'analyse ordinaire incluant la pulsion et le travail ordinaire dans la situation transférentielle, que je considère à vrai dire toujours comme allant de soi, sachant qu'il est sans objet de chercher à découvrir de nouvelles choses si on oublie les anciennes. »

Lettre à Hanna Ries, 27 nov. 1953, in Lettres vives, p. 94.

« ...nous essayons tous d'exprimer la même chose, à ceci près que j'ai une manière irritante de les dire dans mon propre langage au lieu d'apprendre à utiliser les termes de la métapsychologie psychanalytique.

J'essaie de trouver pourquoi je me méfie de ces termes si profondément. Est-ce parce qu'ils peuvent donner l'apparence d'une compréhension commune alors qu'une telle compréhension n'existe pas ? Ou est-ce à cause de quelque chose en moi ? Cela peut, bien sûr, être les deux. »

Lettre à Anna Freud, 18 mars 1954, Loc. cit. p. 98.

« Il me semble très difficile d'amener les analystes à considérer la toute première enfance autrement qu'en termes de pulsions et de désirs. »

Lettre à Clifford Scott, le 13 avril 1954, loc. cit. p. 101.

Aussi bien le dire tout de suite: je ne vois pas ce qu'on gagnerait à établir si oui ou non Winnicott adhérait explicitement à la théorie freudienne des pulsions. De toute façon, il est clair que le concept opère dans sa pensée. La question est plutôt de savoir si la place qu'il fait occuper à celui-ci dans le cadre de la tâche pratique est de nature à faciliter ou au contraire à nuire à la conduite de l'analyse. En ce qui me concerne, je n’hésiterai pas à dire que la pensée de Winnicott m’aide à travailler, j'allais 
dire à survivre, comme analyste. Or comme ma pratique d'analyste et ma compréhension de l'ensemble conceptuel qui éclaire mon travail tiennent amplement compte de la notion de pulsion, j'ai à me demander si ces deux aspects de ma pratique clinique et théorique sont congruents ou s'ils dénotent un éclectisme discutable. Je n'ignore par que chacun d'entre nous produit une sorte de « bricolage » théorique avec lequel s'expliquer à soi-même, le plus souvent dans l'après-coup, ce qu'on pratique. Je sais aussi que ce qui se dit en théorie ne correspond pas systématiquement à ce qui se fait en séance. Il ne s'agit pas seulement de l'écart inévitable entre théorie et pratique, tel qu'étudié par Donnet, mais aussi de ces «arrangements », de ces improvisations et expédients que tout analyste, je suppose, est amené à produire dans le cours de sa pratique. Improvisations et arrangements qui dénotent bien la nature particulière de l'aventure analytique; je veux dire par là que cette aventure, comme toute aventure véritable, est ouverte à l'imprévu, qu'elle ne saurait donc être parfaitement balisée par une claire vision théorique de ce qui s'y passe. Ce serait même une monstruosité technique étrangère à l'esprit de l'entreprise analytique et à son éthique, si nous pensions pouvoir tenir sous un contrôle constant et toujours guidé par la théorie ce qui se produit dans le transfert qui noue les deux membres du couple analytique. Il y aurait danger de pensée totalitaire et d'emprise au lieu de l'effort de dégagement et de liberté que représente l'analyse.

Or ce qui est particulièrement intéressant avec Winnicott, c'est que son œuvre est de nature à rendre pensable l'irruption de l'imprévu, du geste spontané et de la création in situ au cours de l'analyse. Théorisation paradoxale, il va de soi, qui pense ses propres limites et la possibilité de son dépassement pratique sans espoir de systématisation en quelque manuel. Cet intervalle de liberté, ces marges un peu floues entre une ferme théorie et une pratique ouverte sur la création, voilà, entre autres choses, ce qui rend pour moi la fréquentation de Winnicott si inspirante, quand bien même 
son alignement conceptuel laisserait trop de « jeu », si on me permet ce clin d'œil. C’est que ce « jeu » me devient au contraire de plus en plus nécessaire dans mon désir, peut-être prétentieux et utopique, de parvenir à dire quelque chose de ne serait-ce que légèrement différent sur mon expérience d'analyste.

Dans cette optique, le texte-phare de Winnicott est pour moi «The Use of an Object and Relating Through Identifications ${ }^{1}$ » qui se base sur une communication faite à New York le 12 novembre 1968, c'est-à-dire pas très longtemps avant sa mort. Pour qui connaît les péripéties entourant cette présentation, ce texte a posé à ses premiers auditeurs le problème précis dont il traite, celui de leur capacité de «survivre » à l'élan créateur-destructeur de Winnicott lui-même. Or on sait que les discutants new-yorkais ont si mal «survécu » que Winnicott lui-même a failli mourir dans la suite immédiate de cette rencontre. Je mentionne cela pour souligner que le texte winnicottien est tellement près de la chose dont il parle qu'il se présente à nous pour ainsi dire « en acte » et non comme simple compte-rendu théorique d'une expérience vécue par lui jadis, ailleurs. À mon avis, c'est la marque d'une psychanalyse vivante, quand, tout comme dans un vrai texte littéraire, l'écrit porte en lui au moins une partie de la charge qui a suscité son écriture et la transmet à ses lecteurs qui, de leur côté, doivent savoir y survivre ${ }^{2}$.

Dans ce sens, le pulsionnel me paraît d'emblée présent chez Winnicott, même quand il ne semble pas intentionnellement visé par lui. Mais ce serait trop simple de se contenter de faire ainsi crédit à Winnicott de son allégeance à la théorie des pulsions. Je crois en effet que le pulsionnel, le sexuel, travaille dans sa pensée en fonction de sa façon particulière de penser la pratique psychanalytique avec la méfiance dont il fait état envers la langue métapsychologique. On pourrait ainsi craindre que

\footnotetext{
${ }^{1}$ In Psychoanalytic Explorations, Édité par Clare Winnicott, Ray Shepherd et Madeleine Davis, Cambridge Mass., Harvard University Press, 1989. Dans cet article, sauf indication contraire, je traduirai moi-même les extraits des textes de Winnicott.

${ }^{2}$ Cf. «Vérité d’un texte », Bulletin de la société psychanalytique de Montréal, vol. 21, nº 3, 2009, p. 40-43.
} 
l'espace potentiel où il se situe puisse se dégrader en relativisme ou en subjectivisme. On peut à mon avis éviter un tel destin à condition de garder bien tendue l'écoute au sens auditif comme au sens marin du terme - écoute qui non seulement dénote une éthique de la discussion entre psychanalystes, mais nous fait aussi entendre les échos proprement freudiens de la pensée de Winnicott. Je voudrais ici proposer une telle écoute. L'écoute d'un Winnicott qui ne remplace pas Freud, mais lui ajoute quelque chose comme une nouvelle interface.

\section{Présence du sexuel}

À l'appui de la thèse que Winnicott tient pleinement compte du sexuel, j'invoquerai, outre les lettres citées en épigraphe, des textes comme celui, en apparence très éloigné de la question, intitulé «Contribution de l'observation directe des enfants à la psychanalyse $^{3} »$. Texte de 1957, mais qui reste d'une grande pertinence, surtout aujourd'hui où l'observation directe a beaucoup plus d'adeptes. La finesse dialectique de Winnicott est ici à son apogée. Il distingue, en des termes très simples, entre profond (deep) et précoce $\left(e a r l y^{4}\right)$. Distinction importante qui, comme on le verra, situe assez précisément l'advenue du pulsionnel dans la psyché infantile. Usant implicitement, comme il l'a fait dans d'autres textes, de la notion d'après-coup ${ }^{5}$, Winnicott précise que le profond s'instaure dans un second temps par rapport aux expériences précoces. Il écrit : «Certains concepts ont l'air vrai de mon point de vue quand je pratique l'analyse, mais sonnent faux lorsque je suis en train d'observer des enfants dans ma clinique. » Mais pour qui penserait qu'il oppose là un démenti à la conceptualisation tirée de l'analyse, il ajoute aussitôt que : «[...] l'observation directe est in-

\footnotetext{
${ }^{3}$ On the contribution of Direct Child Observation to Psychoanalysis, in The Maturational Process and the Facilitating Environment, London, Karnac Books, 1990.

${ }^{4}$ La traductrice française a choisi de rendre « early » par «primitif», mais cela pose problème puisque nous pouvons facilement entendre du «profond» dans le «primitif » psychanalytique - pensons ici au «primitif » tel que conçu par Freud dans Totem et Tabou - , tandis que « précoce » exprime tout ce qu’il y a dans « early », sans malentendu possible.

${ }^{5}$ Voir « Fear of Breakdown », in Psychoanalytic Explorations, op. cit. Trad. Fr. « La crainte de l'effondrement », dans le livre éponyme, Paris, Gallimard.
} 
capable de construire par elle-même une psychologie de la première enfance » (p. 114) et il conclut par cette formule : «En deux mots : le petit humain doit faire un bout de chemin à partir du précoce pour avoir la maturité nécessaire à l'être profond. » (Ibid.) Nous tenterons de décrire ce « bout de chemin » mais pour l'instant, mettons en réserve cette distinction entre précoce et profond et tournons-nous vers les commentaires que Winnicott a lui-même faits à son texte sur l'utilisation de l'objet.

Dans un de ces commentaires, daté de janvier 1969 et publié après sa mort, Winnicott met en parallèle les pensées qu'il a développées dans sa fameuse conférence de New York avec le développement de Freud dans « Moïse et le monothéisme ${ }^{6}$ ». Ce rapprochement, étonnant à première vue, se révèle des plus éclairants quant à la position de Winnicott sur la théorie pulsionnelle. Il renvoie en effet explicitement au dernier dualisme freudien. Il serait trop long et hors de notre propos de suivre tous les développements qui figurent dans ce commentaire. J'en souligne les éléments les plus en rapport avec notre thème. Winnicott adhère volontiers à l'opposition — formulée par Empédocle d'Agrigente et citée par Freud à l'appui du dernier dualisme pulsionnel - entre philia, ou tendance à l'agglomération et à l'unité, et neikos, ou tendance au désordre, à la discorde. Comme tant d'autres, Winnicott ne voit aucune raison de rejeter ce dualisme. Sauf que cela ne donne pour lui aucun crédit à une pulsion de mort, et qu'il dit même souhaiter « soulager [Freud] d'avoir à porter à jamais ce fardeau comme un Atlas » ( p. 242). Ce qu'il récuse, c'est l'idée qu'on puisse postuler une pulsion à partir de la tendance de l'organique à retourner vers l'inorganique. C'est une critique que beaucoup d'auteurs contemporains ont été amenés à formuler? Winnicott pense néanmoins qu'avec la pulsion de mort, Freud s'efforçait de

\footnotetext{
${ }^{6}$ Cf. « The Use of an Object in the Context of Moses and Monotheism », Psychoanalytic Explorations, op. cit. p. $240-246$

${ }^{7}$ Cf. Le Chap. VII de mon petit ouvrage Les pulsions, Paris, PUF, Coll. « Que sais-je ? », 2004.
} 
formuler quelque chose de «global» (comprehensive), mais pour lequel lui manquait la leçon qu'allait fournir dans les décennies suivant sa mort l'expérience avec les patients borderline et psychotiques. «Les psychanalystes, écrit-il, allaient apprendre qu'il se passe beaucoup de choses chez les bébés en rapport avec le besoin, tel que distinct du désir et distinct des représentants (pré-génitaux) du ça qui exigent satisfaction. » (p. 242.)

Parlant, toujours dans ce commentaire de 1969, de la destruction de l'objet, il écrit ce qui suit : «On verra que j'essaie de récrire une petite partie de notre théorie. Cette poussée provocante/destructive/agressive/envieuse (Klein), n'est pas un phénomène relevant du principe de plaisir/douleur. Elle n'a rien à voir avec la colère due à la frustration qui est associée au principe de réalité. Elle précède cet ensemble de phénomènes qui sont vrais chez les névrosés mais ne le sont pas chez les psychotiques. » (p. 246). Winnicott, qui récuse la pulsion de mort, admet donc quand même un au-delà, ou un en-deça, du principe de plaisir. Ce qui, au passage, change sensiblement l'idée que l'on peut se faire de sa conception de l'univers des besoins. Loin du « long fleuve tranquille » qu'on semble parfois postuler lorsqu'il est question des besoins vitaux et de l'adaptation, il y voit plutôt des poussées potentiellement destructrices du seul fait, pour le bébé, de vivre, de respirer : « À cette étape d'importance vitale, la vivacité destructive (feu, air ou autre) de l'individu est simplement un symptôme du fait d'être vivant... » (p. 239). Vitalisme au lieu du pulsionnel ? Je crois au contraire que la « vivacité destructive » dont il est question, non seulement préfigure le pulsionnel mais en prépare le terrain, selon une logique que je tenterai de mettre en évidence.

En attendant, si la question du besoin n'est pas, chez Winnicott, un vestige inutilisable pour la psychanalyse, mais se pose en termes de destruction, cela change totalement la donne. Et les racines freudiennes ne sont pas loin si l'on se souvient 
que Freud a, pour un bref moment de son texte de 1919, fait s'équivaloir pulsions du moi (ou d'autoconservation) et pulsions de mort ${ }^{8}$. Son repentir ultérieur, qui lui a fait a ranger l'autoconservation sous le parapluie d'Eros, est objet de discussions encore de nos jours ${ }^{9}$. Revenant à Winnicott, peut-être trouvons-nous ici une première approximation de ce qui mènera du précoce au profond, si nous posons que le profond correspond à l'instauration du pulsionnel sexuel et du principe de plaisir dans la vie psychique, tandis que le précoce fonctionne sous l'égide du besoin, entendu cependant comme une forme de l'au-delà du principe de plaisir et donc apparenté de quelque façon avec le pulsionnel de mort freudien, celui que Freud lui-même a fait correspondre, ne serait-ce que brièvement, aux pulsions du moi ou d'auto-conservation. Winnicott ne raisonne pas explicitement en ces termes, mais ce retour inopiné à un au-delà du principe de plaisir qui logerait dans le domaine du besoin devrait retenir notre attention. Il est en effet peut-être trop facile de dichotomiser le domaine des pulsions et celui des besoins. Sans confondre ces deux registres, on peut avancer que le besoin humain doit bien avoir en lui, du fait de l'hilflösigkeit du nouveau-né, une affinité particulière avec le pulsionnel, affinité qui nous autorise à intégrer, comme le fait Winnicott, le plan du besoin dans le champ psychanalytique lui-même. Freud ne raisonnait pas autrement, dans les Trois essais. Ainsi, la « poussée provocatrice/destructrice » que Winnicott insère dans l'échange avec l'objet, ne serait-elle pas une forme précoce du pulsionnel à venir ? J'oserais même avancer que nous trouvons là, à plus fort grossissement, le champ où se joue la séduction originaire, champ qui est d'habitude sinon exclu, du moins posé comme simple toile de fond (l'attachement, p.ex.) et très peu théorisé psychanalytiquement. Or, la pensée winnicottienne m’amène à poser que l'incapacité de survivre que démontre l'objet exerçant des représailles correspond assez bien à l'intromis-

\footnotetext{
${ }^{8} \mathrm{~S}$. Freud, Au-delà du principe de plaisir, OCF-P, v. XV, p. 311, puis le repentir en p. 326. Voir aussi la longue note en p. 335.

${ }^{9}$ Michel de M’Uzan, par exemple, regrette que Freud ait renoncé à cette conception.
} 
sion dont parle Laplanche, variante violente de la séduction. L'angle d'approche est différent, mais la description me parait sans contradiction avec cette théorie. Avec l'avantage de décrire plus finement la mécanique relationnelle telle que vécue du point de vue de l'enfant, rendant compte avec plus de précision de l'expérience de l'intromission. Il est probable que Laplanche n'admettrait pas ce rapprochement $^{10}$, mais je le crois néanmoins légitime et utile, comme je tenterai de le montrer.

\section{L'irruption paternelle de l'unité et la fracture sexuelle}

Dans le commentaire de 1969 déjà cité, Winnicott nous surprend en invoquant la recherche amorcée par Freud, dans son Moïse, sur la question du père. Un père dont le rôle spécifique se révèle, écrit Winnicott, «à mesure que le bébé va d'un renforcement du moi dû à l'apport du moi de la mère vers l'acquisition d'une identité propre - c'est-à-dire que, à mesure que la tendance innée à l'intégration fait progresser l'enfant dans l'environnement good-enough ou environnement moyen prévisible - la tierce personne joue ou me semble jouer un grand rôle. » (p. 242) On voit ici surgir implicitement le père comme tiers séparateur entre l'enfant et la mère. Winnicott formulera la chose ainsi : «Le père peut, ou non, avoir été un substitut maternel, mais il commence parfois à être éprouvé comme jouant un rôle différent, et je propose que c'est alors que le bébé est en mesure d'utiliser le père comme modèle (blueprint) pour sa propre intégration lorsque par moments il devient unifié. »

Le père fait donc son entrée dans l'univers de l'enfant en tant que prototype de l'unité, mais je crois utile de considérer que ce faisant il introduit aussi la différence entre l'unité que constituait l'ensemble mère-enfant et l'unité nouvelle, qui est celle du père

\footnotetext{
${ }^{10}$ Laplanche a récusé, dans son séminaire, ce que j’ai avancé là-dessus dans un texte publié au Royaume-Uni, «Laplanche and Winnicott meet... and survive», in (sous la direction de Lesley Caldwell) Sex and sexuality: winnicottian perspectives, London, Karnack Books, 2000.
} 
seul et qui sera aussi celle de l'enfant lui-même. On peut d'ailleurs poser que c'est au point de rencontre (ou de séparation) entre ces deux formes de l'unité que surgit le mouvement pulsionnel.

Winnicott aligne ses remarques à propos du père sur celles de Freud parlant de l'unification apportée par le monothéisme, qui est aussi une universalisation. : «Un dieu, un monothéisme, et non un dieu pour moi et un autre dieu pour toi », écrit Winnicott. Mais ce dieu unitaire qu'est le père sera bientôt « doté d'un objet partiel significatif ». Et avec cet objet partiel, il est clair que nous nous trouvons maintenant dans la sphère du sexuel, du pulsionnel. On voit ainsi que l'attention de Winnicott est centrée non sur le seul monde des besoins, mais sur la brèche dynamique, sur ce qui travaille entre le plan du besoin et le plan du sexuel. Selon la piste que nous suivons ici, le pulsionnel sexuel est inauguré chez Winnicott par la fracture du père unitaire, fracture qui survient après l'accès de l'infans à son unité et à son identité propre - à sa singularité. Toute la question sera de penser comment s'opère cette fracture par où s'introduit le pulsionnel. Cela nous donnera l'occasion de voir la théorie de la séduction apporter à son tour quelque lumière sur le modèle winnicottien.

L'apparition du père unitaire et de son « objet partiel significatif » permet de préciser davantage ce que Winnicott entend par « destruction » dans le texte sur l'utilisation de l'objet ${ }^{11}$. L'unité et l'identité acquises à l'aide du prototype paternel entre en effet dans un mouvement dialectique où, pour l'enfant, assumer sa singularité (en pensant par soi-même, par exemple) implique automatiquement une " destruction » qui n'est pas anéantissement, mais fractionnement de l'objet en personne « totale » d'une part, et objet partiel ou objet du désir ou de la pulsion d'autre part ; objet, aussi, du fantasme. Il va de soi qu'après l'identification de l'enfant au prototype unitaire du

\footnotetext{
${ }^{11}$ J'ai souligné dans un autre article le fait que Winnicott ne dit pas ce qu'il entend par «destruction», et j'ai proposé que c'était en toute logique avec sa pensée sur le sujet (cf. D. Scarfone, "À quoi œuvre la psychanalyse ? ", Libres cahiers pour la psychanalyse, $n^{\circ}$ 9, 2004, p. 109-123.) Rien ne nous interdit cependant de faire état de notre interprétation.
} 
père, l'objet à détruire, c'est-à-dire, ici, à décomposer en total et partiel, ce n'est pas le père uniquement, mais toute personne significative. L'apparition du sentiment et du concept d'unité a désormais rendu pensable et désirable le partiel, là où il n'y avait auparavant qu'un « tout».

Que l'objet soit « toujours en train d'être détruit en fantasme », comme l'écrit Winnicott dans "The use of an Object», cela peut donc aussi s'entendre en clef sexuelle : détruire en fantasme peut signifier détruire par le fantasme ; la « destruction » (qui, précise Winnicott, ne comporte aucune agressivité) de l'objet total résultant du simple fait de désirer et de fantasmer en lui l'objet partiel. Il sera dès lors attendu de l'objet externe qu'il se laisse utiliser, dans le sens de se prêter au " prélèvement » de l'objet fantasmatique, c'est-à-dire de laisser l'enfant développer son univers de désir, son univers fantasmatique, sans exercer de représailles. C'est à ce point que l'on peut le mieux raccorder la pensée Winnicott avec la théorie de la séduction de Laplanche. Il me semble en effet qu'une des formes que peuvent prendre les "représailles » en question, c'est pour l'adulte d'entrer dans un rapport de séduction perverse et d'abolir ainsi violemment la distance entre objet du besoin (objet pourvoyeur du soin, objet du «courant tendre ») et l'objet du désir ou du fantasme de l'enfant, menaçant du même coup l'unité individuelle acquise par cet enfant, du fait de la soumission qui est exigée de lui. Si on croit entendre là un écho de la confusion de langues ferenczienne, ce n'est pas un hasard. Dans la langue de Winnicott, on peut ajouter que l'espace transitionnel dans lequel l'objet trouvé-détruit est appelé à évoluer est littéralement traumatisé, c'est-à-dire déchiré, écrasé par cette intrusion réelle, trop réelle, d'un objet violemment incapable de survivre.

La référence à Laplanche ne découle cependant pas du simple plaisir de trouver des correspondances. Elle nous permettra d'aplanir certaines difficultés inhérentes au 
modèle winnicottien. Nous avons en effet donné à l'enfant le rôle de décomposer l'unité paternelle (et ensuite maternelle) pour y désirer l'objet partiel. On peut légitimement se demander par quelle magie s'opérerait cette découpe, ce fractionnement. À ce sujet, il me semble évident que Winnicott reste prisonnier d'une conception « instinctuelle » du pulsionnel et d'une description un peu trop empirique du cours des événements. Nous verrons maintenant qu'après avoir jeté quelque lumière sur le moment de la séduction, la pensée de Winnicott pourra à son tour être éclairée par la théorie de la séduction.

Quand Winnicott souligne que le début de la vie ne lui apparaît pas tout descriptible en termes de pulsions et de désirs, il me semble ouvrir exactement l'espace où intervient la séduction originaire. À tout le moins, il y a accord entre les deux théories sur le fait que l'enfant n'arrive pas au monde avec un appareil pulsionnel préformé et prêt à agir. La séduction originaire, comme on sait, opère à l'insu des séducteurs euxmêmes qui, du fait de leur sexuel inconscient, émettent de messages véhiculant un excès. Qu'en est-il de cet excès ? Ne serait-ce pas ce qui provoque et justifie la décomposition partielle, opérée par l'enfant, de l'unité introduite par le père ? Si oui, comment?

Par rapport à l'expérience de l'unité individuelle, on peut dire que l'unité de l'adulte est nécessairement amenée à vaciller du fait d'être habitée par le sexuel refoulé. L'objet se voudrait seulement tendre, mais il est habité par du sexuel inconscient. L'enfant ne capte pas normalement le «sexuel» en tant que tel ; mais il peut très bien sentir les effets du vacillement identitaire que ce refoulé provoque chez l'adulte. Ce vacillement, je propose que c'est la forme sous laquelle se présente l'excès que capte l'enfant. L'expérience d'un « relating » tendre est perturbée par ce vacillement. La « destruction » opérée par l'enfant serait ainsi la réponse à la perception obscure de ce vacillement à travers lequel il sent qu'il y a plus et autre chose dans, devant ou der- 
rière l'image unitaire de l'adulte. Cela impose à l'enfant la tâche de penser par luimême cette énigmatique oscillation, travail herméneutique infini qui fait de l'enfant — de chacun de nous - un traducteur-destructeur de la belle et mystifiante unité de l'adulte.

En s'en tenant au père winnicottien, on peut formuler la même idée autrement, en disant que l'énigme réside dans la différence éprouvée entre le père-simple substitut maternel et le père unitaire, symbolique. Cette différence interne à l'unité faisant message énigmatique, la destruction se conçoit comme ceci : ayant introduit l'unité et par là un idéal de cohérence et de non-contradiction (" un seul dieu pour tous »), le père se verra « détruire » par l'effort qu'il aura lui-même suscité chez l'enfant du fait de son «vacillement ». L'effort de parvenir à UN sens, à UNE version unitaire du monde sera voué à l'échec du fait de l'impact du pulsionnel refoulé de l'adulte. Échec nécessaire, faut-il ajouter, sans quoi ce serait l'adhésion sans réserve au discours de l'autre, de l'adulte infaillible; mais échec qui, si l'objet ne survit pas, peut décourager la recherche infantile et la réorienter dans le sens du retour à la seule unité effective qui ait existé : l'indifférenciation mère-enfant. Rappelons que ce mouvement de retour à un état antérieur correspond à ce que Freud assigne, dans son dernier dualisme, au pulsionnel de vie comme de mort ${ }^{12}$. Mais ici, nous dirons avec Winnicott : nul besoin de retour à l'inorganique. Le retour à cet impensable d'avant la différenciation, Hans Loewald a signalé que cela a toujours été la visée des pulsions, dans toutes les versions de la théorie freudienne ${ }^{13}$. Retrouver l'objet primordial, en effet, ce n'est pas pour en jouir en tant que sujet, ce n'est pas pour «l'utiliser », c'est pour s'y dissoudre, pour abolir le manque. Toutes les pulsions, y compris la pulsion sexuelle du premier dualisme freudien, sont en ce sens « conservatrices » et visent un retour. Se-

\footnotetext{
${ }^{12} \mathrm{~S}$. Freud, Au-delà du principe de plaisir, op. cit.

${ }^{13}$ Cf. Hans Loewald, «Ego and reality » (1949), ainsi que On Motivation and Instinct Theory (1971), tous deux in The Essential Loewald, Hagerstown MD, The University Publishing Group, 2000, p. 3-20.
} 
lon Loewald, « un courant libidinal, [c'est ce qui] surgit entre l'infans et la mère, dans une poussée visant à rétablir l'unité originelle ${ }^{14}$. » Par conséquent, le sexuel n'est pas à vrai-dire quelque chose qui s'introduit comme une " chose » dans la psyché de l'enfant. La libido est plutôt, comme le suggérait Lacan, " couleur de vide, suspendue dans la lumière d'un béance ${ }^{15} »$; non une entité positive, mais un courant qui s'installe entre l'enfant et l'objet-perdu-et-à-retrouver ; objet dont les retrouvailles, si elles étaient possibles, ramèneraient du profond au précoce, en direction de l'indifférencié.

Dans cette optique, l'implantation du sexuel dont parle Laplanche se laisse concevoir comme moment particulier du courant libidinal tel que conçu dans ce contexte winnicottien. Ce moment serait précisément celui de la mutation et complexification du précoce en profond, selon les termes que nous avions mis en réserve tout à l'heure. Le précoce correspondrait à un moment préalable, moment où la visée libidinale d'origine, la tendance au retour à l'unité primordiale, emprunte la voie de sa réalisation directe, favorisée par la très réelle préoccupation maternelle primaire et la satisfaction effective, quoiqu'imparfaite, des besoins de l'enfant.

Le second moment s'ouvre, lui, avec la présentation de l'unité paternelle : l'enfant se sent maintenant, même si par moments seulement, lui-même " un », sans pour autant être uni à la mère. L'unité individuelle se substitue ainsi à l'unité primordiale mère-enfant que cherchait à rétablir le courant libidinal primaire. L'unité nouvelle, résultat de l'identification à l'unité présentée par le père, on a vu qu'elle ne tarde pas à se fracturer sous l'effet de l'enquête créatrice-destructrice de l'enfant, quand celui-ci se met à penser la différence introduite par ce tiers ${ }^{16}$. Mais nous disions que Winnicott s'en tient peut-être ici à une description trop empirique. En effet, on ne saurait négliger le

\footnotetext{
${ }^{14}$ Loewald, « Ego and reality » Op. Cit. p.6.

${ }^{15}$ J. Lacan, « Du "trieb” de Freud et du désir du psychanalyste », in Écrits, Paris, Seuil, 1966, p. 851.

${ }^{16}$ On retrouve ainsi la thèse freudienne du « Moïse » selon laquelle le père est du côté du «progrès spirituel».
} 
fait que la perception du vacillement identitaire ne se produit pas exclusivement dans le rapport au père. La différence s'éprouve aussi bien avec la mère, celle-ci étant tout autant porteuse d'un sexuel inconscient qui contamine ses «messages». Nous compliquerons donc un tout petit peu le tableau peint par Winnicott en posant que l'apparition du père en tant que tiers (au lieu du substitut maternel) introduit l'expérience de l'unité non par magie, mais parce que ce tiers force l'enfant — qui jusque là alternait entre un sentiment passager d'unité individuelle et un retour à l'union avec la mère - à éprouver tous les objets comme individus distincts. Ce tiers, en effet, fait du même coup sentir que la mère elle-même désire ailleurs. Pour l'enfant, le retour à l'union avec la mère n'est plus aussi aisé qu'avant, d'autant plus que le père, agent d'une expérience nouvelle, devient lui-même un pôle d'attraction qui le distrait de son rapport libidinal plus ou moins exclusif à la mère. Le vacillement dû au pulsionnel chez les adultes fait maintenant émerger des objets de désir qui sont forcément conçus sur le modèle des objets partiels, objets qui avaient été jusque là concrètement prélevés sur le corps de la mère mais non vécus ou pensés comme partiels. La survenue du père cristallise en quelque sorte la différence plus confusément pressentie, sinon éprouvée, dans le rapport à la mère, entre courant tendre lié au besoin et courant « véhément » du sexuel, entre relation à la personne totale et irruption de l'objet partiel, objet de désir.

Nous voici donc capables, tout en usant d'un idiome winnicottien, d'identifier non pas un, mais deux moments sexuels. D'une part un courant libidinal que nous dirons " précoce » : c'est un sexuel qui, dans les faits, est par moments presque harmonieusement réalisé vu l'adéquation optimale entre l'enfant et son environnement 
maternel ${ }^{17}$. D'autre part, un sexuel «profond », résultat d'une reprise créatrice-destructrice consécutive à l'expérience de l'unité, expérience qui advient dans le cadre de la séduction et qui correspond au pulsionnel proprement dit de la théorie classique. Pulsionnel qui, contrairement au courant libidinal primaire, conduit du côté de la complexification et de la psychisation. Notons en passant que cette double caractérisation du sexuel nous permet de résoudre en partie le problème de savoir si les pulsions sont des moteurs de progrès (Freud, version 1915) ou sont essentiellement conservatrices (version 1919). On peut affirmer qu'elles sont les deux à la fois puisque si la libido «précoce» est d'emblée dirigée vers le retour à l'indifférencié, le mouvement progressif du pulsionnel «profond», tout tourné vers la recherche d'un objet dans le monde extérieur, vise lui aussi, en définitive, le retour à l'unité, bien que par des voies indirectes, long chemin sur lequel il rencontre des objets à « utiliser ». La visée ultime des deux régimes sexuels est donc à la limite la même : rétablir l'unité primordiale, dans le premier cas à travers le seule « relating » à peine émergé de l'indifférenciation, dans le second cas, à travers une utilisation de l'objet, utilisation qui suppose l'existence d'un espace d'expérience distinct, bien à soi. Si, pour l'enfant ayant fait l'expérience de l'unité individuelle, cette visée devait se réaliser réellement, elle exigerait du même coup qu'il renonce à penser par lui-même, qu'il renonce par conséquent à sa destructivité pour s'engager sur le chemin du retour vers l'unité indifférenciée. Mais, comme on sait, il n'y a pas de retour. L'objet qui ne survit pas, c'est donc celui qui impose à l'enfant ce renoncement et ce-faisant le laisse, non dans une béatifique union avec l'objet, mais sous le diktat de son message intraduisible, dans la pire solitude qui soit.

\section{Dominique Scarfone}

Septembre 2010

\footnotetext{
${ }^{17}$ Sexuel implicite dans le Winnicott du besoin ; présent anonymement, comme l'air qu'on respire et en rapport avec ce que dit Mereleau-Ponty (L'Institution.La Passivité. Notes de cours au Collège de France 1954-55.Paris, Belin, 2003.) de la nature sexuelle des rêves ; ceux-ci ne parlent pas du sexuel, ils sont sexuels. " La tâche st moins de donner clef sexuelle, transcription sexuelle en pensée conventionnelle que de faire retrouver la sursignification du sexuel dans la vie préobjective » (p. 212); « ...la censure consistant dans le refus de notre passivité et de sa grande pourvoyeuse : la sexualité. »
} 